\title{
Urgences
}

\section{Le cas de la poésie mise en discours dans un roman : Le nez qui voque de Réjean Ducharme}

\section{Richard Duchaine, Louise Milot et Dominique Thibault}

Numéro 28, mai 1990

Le roman comme poétique

URI : https://id.erudit.org/iderudit/025584ar

DOI : https://doi.org/10.7202/025584ar

Aller au sommaire du numéro

Éditeur(s)

Urgences

ISSN

0226-9554 (imprimé)

1927-3924 (numérique)

Découvrir la revue

Citer cet article

Duchaine, R., Milot, L. \& Thibault, D. (1990). Le cas de la poésie mise en discours dans un roman : Le nez qui voque de Réjean Ducharme. Urgences, (28), 7-19.

https://doi.org/10.7202/025584ar d'utilisation que vous pouvez consulter en ligne. 


\title{
Le cas de la poésie mise en discours dans un roman: Le nez qui voque de Réjean Ducharme
}

\author{
Richard Duchaine, Louise Milot \\ et Dominique Thibault
}

Qu'un roman puisse constituer en définitive une poétique ouvre une avenue de recherche tout à fait fructueuse, si tant est que l'on donne à "poétique " le sens général d'un discours ou d'un point de vue sur l'écriture. En regard des objectifs du présent numéro, toutefois, la question est de savoir si le terme "poétique» peut prendre le sens plus spécifique d'un discours sur la poésie dans le cas d'œuvres romanesques qui paraissent faire explicitement référence à ce genre. Répondre oui à cette question implique certes une prise de position concernant la lecture, voire une définition de la lecture selon laquelle les réseaux figuratifs d'un roman donné produiraient un à un et en eux-mêmes un discours.

À l'intérieur de la vaste problématique des rapports du roman et de la poésie, c'est cette question dont nous voulons interroger la pertinence. Nous laissons à la stylistique ou à la rhétorique tout le champ de la forme ou du style poétiques d'un roman; et aux plus courageux que nous, celui de l'intertextualité, qui met davantage en valeur les ressources de l'érudition que celles de la dynamique textuelle. Seule nous retiendra la mise en scène dans le texte des figures explicites de la poésie.

Le nez qui voque de Réjean Ducharme ${ }^{1}$, qui nous servira d'exemple, est justement un de ces romans auxquels la critique a reconnu assez unanimement, dès sa parution, une composante poétique au sens strict. Le texte semble renvoyer, comme à des modèles, à des poètes connus (Rimbaud et Nelligan, notamment); le personnage central de Mille Milles réclame désespérément pour lui-même la profession de poète (p. 166); de nombreux poèmes jalonnent le cours du texte; sans parler de l'omniprésence des jeux de mots de toutes

1. Réjean Ducharme, Le nez qui voque, Paris, Gallimard, 1967. Les indications de pages entre parenthèses renverront à cette édition 
8

sortes ${ }^{2}$ et sans aucun doute des nombreux collages ou pastiches de poèmes dissimulés dans le récit ${ }^{3}$. Pas étonnant que ce second roman de Ducharme ait été qualifié tout à la fois de "longue et tumultueuse prosopopée lyrique » et de * poème chaotique 4 .

Nous essaierons pourtant de démontrer que les figures de la poésie sollicitées et actualisées par un texte de prose n'appellent pas une interprétation ponctuelle. S'il peut être intéressant et opératoire de les isoler pour les besoins de l'analyse ${ }^{5}$, ces figures n'échappent pas à leur juste valeur de figures parmi d'autres dans l'ensemble du déploiement figuratif. Ainsi, en mettant en scène un autre genre - ici la poésie -, le roman ne se trouverait pas nécessairement à produire, au sujet de celui-ci, un discours.

Afin de faire voir à quels enjeux une telle façon de voir mène la lecture et l'analyse, nous procéderons en trois temps. $\grave{A}$ titre exploratoire et en guise d'exemple liminaire, nous confronterons notre problématique à un segment que nous nous croyons autorisés à isoler, soit le prologue intratextuel (p. 7) qui précède la "Préface" du roman (p. 8). Nous lirons ensuite dans cette foulée deux types de manifestations poétiques du roman: d'abord, certains commentaires de la poésie intégrés à la prose; puis la présence de poèmes proprement dits. Nous devrions pouvoir ainsi (re)tracer un cheminement.

\section{Le prologue: à propos de Nelligan}

L'ouverture du Nez qui voque par le moyen d'une citation de Soir d'hiver de Nelligan est certainement pour quelque chose dans le halo poétique du roman 6 . Certains critiques ont remarqué les citations cavalières qui suivent ${ }^{7}$, ou encore la dernière phrase, premier (et excellent) exemple du savoir-

2 Voir l'article de Bernard Dupriez, a Ducharme et des ficelles , Voix et images du pays, Montréal, vol. V, 1972, p. 164-185.

3 Monique Genuist en a révélé quelques occurrences dans $\propto$ Mille Milles et la Femme dans $L e$ nez qui voque ", Atlantis, vol. II, n² 2, printemps 1977, p. 56-63.

4 Alain Clerval, « Sur Dieu et les femmes ", La quinzaine littéraire, 15 au 30 juin 1967, p. 10.

5 Nous menons présentement des recherches en ce sens, au CRELIQ de l'Université Laval, sur l'inscription des figures de l'écrit dans des romans québécois de 1837 a 1960.

6 Voir le début de l'article déjá cité de Monique Genuist.

7 Bernard Dupriez y voit (article cité p. 166) « un chapelet désordonné de simili-citations 2 . 
faire linguistique caractéristique de l'écriture du roman ${ }^{8}$. Or, cette page d'ouverture requiert à notre avis d'être lue en entier et en simultanéité, seul moyen d'y capter la fonction des quatre vers postés à son entrée 9 .

Nous proposons de diviser le prologue en quatre parties:

1. La citation de Soir d'hiver de Nelligan (lignes 1-5);

2. La suite de citations tronquées de personnages célèbres et majoritairement d'écrivains (lignes 6-14);

3. Les quatre phrases attribuees deux à deux à Iberville et à Léandre Ducharme (lignes 15-21);

4. Le groupe des quatre phrases finales, d'un auteur imaginaire (lignes 22-24).

1. Indépendamment de l'intérêt qu'il peut y avoir à scruter l'incipit d'un texte, celui-ci est loin d'avoir une importance stratégique comparable à celle de ses segments terminaux. La sémiotique, pour ne nommer qu'elle, a bien établi que le point de départ d'un discours est à lire comme un contenu inversé, par rapport au contenu (dit posé) qui finit par être établi au moment de son achèvement ${ }^{10}$. Aussi les vers de Nelligan, indispensables ici, de toute évidence, au démarrage du récit, doivent-ils être mis en perspective: il serait risqué d'assimiler leur mise en discours à une quelconque profession de foi quant à l'importance de la poésie, ou de la poésie de Nelligan. Il faut voir dans ces références poétiques initiales la phase de manipulation ${ }^{11}$, l'indication, en quelque sorte, des enjeux de ce discours.

\footnotetext{
$8 \quad$ lbid., p. 167.

9 Dans un ouvrage récent, Françoise Laurent insiste sur cette préface, dans laquelle elle voit $\propto$ un champ magnétique d'où partent et où aboutissent toutes les lignes de force de l'œuvre romanesque de Ducharme s: Mais le commentaire qu'elle en fait est très libre et tient peu compte des contraintes de la dynamique textuelle. Voir L'couvre romanesque de Réjean Ducharme, coll. « Approches", Montréal, Fides, 1988, p. 57-61.

10 Voir un article classique d'A.J. Greimas sur le sujet dans Communications 8: “ Pour une théorie de l'interprétation du récit mythique *. Cette problématique est reprise par Louise Milot dans "Comment lire un texte de fiction ", Le risque de lire, Québec, Nuit blanche éditeur, 1988, en particulier, p. 15-17.

11 Nous utilisons ici le découpage de la séquence canonique de la narrativité, dont les quatre phases sont définies par A.J. Greimas et J. Courtés, Sémiotique. Dictionnaire raisonné de la théorie du langage, Paris, Hachette Universite, 1979, p. 221-222, 252-255, 270-272, 320-321. L'application en est faite ici au niveau de l'énonciation, non de l'énoncé; pour la différence entre les deux, voir Louise Milot, « Sémiotique anthropomorphe vs sémiotique textuelle", Protée, Chicoutimi, vol. 11, $n^{\circ} 3$, automne 1983, p. 4-18.
} 
10

Il devient pertinent, dans ce contexte, que le début du poème soit cité "de mémoire" (ligne 4). On est ainsi prévenu qu'il y a risque d'erreur, ce qui est en effet le cas. Dans le texte original, le premier vers ("Ah comme la neige a neigé ") est repris en refrain au centre de la strophe qui en compte cinq; et le second se lit: «Ma vitre est un jardin de givre » et non pas, comme ici, «Mon cœur est consumé de givre * ${ }^{12}$. La répétition attendue au troisième vers, pourtant essentielle au rythme du poème, est occultée par la mémoire au profit d'une communication immédiate; alors que la métaphore de la "vitre * contiguë au « jardin de givre ", qui passait par un objet, glisse vers le point de vue plus subjectif d'un état d'âme ( Mon cœur»). Comme si la modification allait vers l'expression plus directe du sujet, au détriment d'un travail d'objectivation par le langage: le caractère travaillé et à la limite intouchable propre au poème comme genre ne peut qu'en être atteint.

2. La brèche est ouverte par où s'engouffreront, immédiatement après, la série désinvolte des "Ah", "Oh», «Je me...", "Sur la...", "Il fait ", etc., qui réduit le poids sémantique des éventuelles citations véritables de Colette, Barrès, Mauriac, Gide, etc., ou à d'extrêmes débuts de phrases, ou à des structures linguistiques minimales. L'hypothèse est de voir dans cette apparente provocation la phase de compétence du texte: l'initiative d'un (nouveau) savoir-faire linguistique, seulement suggérée dans la phase antérieure, se place ici d'emblée en situation de (re)commencement. Marquant avec force, d'une part, la présence d'une pléiade de grands prosateurs (et non plus d'un poète), et ramenant d'autre part le contenu de leurs citations à une sorte d'abc de la prose sans commune mesure avec la poésie (nelliganienne), le Sujet d'énonciation fait certes la preuve qu'un nouveau départ est possible. Aussi estil significatif de voir réutilisée, dans une banale répétition, la contrainte rythmique même qui avait été contournée précédemment: le «Ah» de Colette (ligne 6) est répercuté par celui de Platon (ligne 9), puis par celui de George Sand (ligne 13).

3. A partir de la ligne 15 , la cavalcade s'arrête et le texte bascule, le contenu sémantique refaisant progressivement surface dans des phrases d'abord incomplètes (lignes 15-17),

12 Émile Nelligan, Poésies complètes. 1896-1899, * Collection du nénuphar ", Montréal, Fides, 1952, p. 82. 
puis complètes (lignes 18-21), assumées par des auteurs non littéraires, qui sont des auteurs incomplètement puis complètement d'ici: Iberville (acteur de transition), et surtout ce fameux Léandre Ducharme. Pour ne pas être un parfait inconnu ${ }^{13}$, celui-ci est certes plus embarrassant à identifier que Musset ou Colette, mais son patronyme le rapproche suffisamment de la situation d'énonciation, déjà suggérée par la présence de l'uauteur» (ligne 20), pour que s'enclenche la transformation; que s'y passera-t-il donc?

Les deux propositions attribuées (et d'ailleurs réellement empruntées ${ }^{14}$ ) à Léandre Ducharme sont banales, naïves même, chacune à sa façon. La première accuse une redondance (un baleinier qui pêche des baleines); l'autre cède au paradoxe: on sollicite habituellement l'indulgence pour la mauvaise qualité d'une production, non pour sa qualité. Or, ces deux occurrences gagnent à être lues dans le prolongement de la phase de manipulation à laquelle elles font écho, très exactement dans le prolongement des lieux où le poème de Nelligan avait été modifié: ses deux premiers vers. On constate alors que la première phrase de Léandre Ducharme reprend, dans une prose qui la banalise, la répétition de neige et de neige (baleine/baleinier), alors que la mise en contiguité paradoxale de l'indulgence et de la qualité renvoie à l'oxymore du cœur consumé de givre. Ainsi, une transformation a bien eu lieu, et nous pouvons parler de performance: aux lignes 18 à 21 , dans les voies mêmes tracées par la mémoire défaillante du narrateur - donc dans des voies très subjectives -, un certain Ducharme, inconnu jusqu'ici du lecteur, se profile en lieu et place de ce Nelligan (et autres auteurs connus) dont le narrateur ne s'était souvenu des textes qu'imparfaitement.

4. La situation ainsi retournée, le résultat final peut advenir, et il risque de constituer une sanction positive. C'est, cette fois, du (Réjean) Ducharme grand cru: «Le beau n'est pas nécessairement difficile à faire. Le beau n'est pas nécessaire. Le beau n'est pas. Le beau nez !» (lignes 22-24) Comment

13 Un Louis-Léandre Ducharme a fait la rébellion de 1837-1838 et a publié en 1845 son Journal d'un exile politique aux terres australes; voir Françoise Laurent, op. cit., p. 59.

14 Louis-Léandre Ducharme, Journal d'un exile politique aux terres australes, Montréal, F. Cinq-Mars, 1845 [Réédition-Québec, 1968], p. 17 et p. 3, avec de légères modifications. 
ne pas voir dans ce quatuor final une reprise des quatre vers de Nelligan, mais reformulés à la façon de cet auteur imaginaire assez facile à identifier, et qui pose désormais son anonymat de Sujet d'énonciation contre l'identité de Colette, de Barrès, etc., et de Nelligan bien sûr, qui pose du même coup le caractère brillamment travaillé de ses quatre phrases contre le dérisoire de leurs " $\mathrm{Oh}$ * et de leurs * $\mathrm{Ah}$ ", et surtout comme un "négatif " prosaïque de Soir d'hiver. Non seulement, en effet, dans les trois premières propositions de l'auteur imaginaire, le beau, l'essence de la poésie, est-il traité dysphoriquement - a) il n'est pas difficile à faire; b) pas nécessaire; c) inexistant - mais le jeu de mots de la fin marque un saut qualitatif par lequel le substantif «beau», ravalé au rang d'adjectif d'un nouveau substantif, se trouve à produire, à partir de ce qui était un beau poème de Nelligan, cet équivalent trivial méconnaissable qu'est un beau nez.

La rapidité de ce commentaire ne devrait pas occulter l'essentiel: la référence à tel poème ou à la poésie, dans un texte, ne fait que révéler, en définitive, le répertoire des figures potentielles dont dispose un auteur. On voit bien ici que l'extrait de Nelligan s'inscrit dans un discours plus général que lui, et au sujet duquel nous n'anticiperons pas avant de voir si notre hypothèse peut se déporter à d'autres occurrences de la poésie dans ce roman.

\section{La poésie lue par la prose}

Le narrateur du Nez qui voque a ainsi toutes les allures d'un homme de lettres. Par le biais du personnage principal, Mille Milles, non seulement pratique-t-il la poésie, mais aussi la critique littéraire et la correction du français. Les chapitres 9 et 10 (p. 34-37), qui encadrent une première création poétique (p. 35-36), paraissent de ce point de vue un lieu d'observation privilégié: en alternance avec des considérations érotico-linguistiques, Mille Milles y analyse deux alexandrins. Débutant après le second quatrain du chapitre 9 , et se terminant avec le chapitre 10 (p. 36-37), la séquence qui nous intéresse est construite comme le quatrain aux rimes croisées qui la précède: la première et la troisième partie sont consacrées à des questions "linguistiques " (accord du mot *hostie" et polysémie du mot «règles»); alors que la seconde et la quatrième analysent les deux alexandrins. 
Mille Milles fournit d'abord différents exemples d'emploi du mot "hostie», pour ensuite en consigner la loi générale. Tout à fait correcte dans sa forme, la démonstration comporte pourtant deux incongruités. En recatégorisant linguistiquement "hostie" (dans les exemples, il a statut d'adverbe et non de substantif), le narrateur soumet à la norme une manifestation marginale de la langue (un sacre), surtout de la langue écrite. La cohérence du discours grammatical est en outre parodiée, étant non opératoire: censé varier «en genre et en nombre avec le mot auquel il se rapporte, quand il n'est pas employé adverbialement" (p. 36), le mot «hostie» ne varie bien entendu jamais dans aucun des exemples, puisqu'il y est adverbe.

La leçon se poursuit par la lecture critique d'un alexandrin jusqu'ici mal reçu et dont Mille Milles ne donne pas la référence, quoiqu'il semble la connaître: Où l'insecte doré sur le tronc géant rôde. Il en apprécie l'inversion littérale doré I rôde, qui pourrait correspondre à une inversion sémantique; et considère tout à fait recevable le sens dénotatif du vers "pour une mouche, un tronc est extrêmement géant "(p. 36).

Ainsi, tout en mettant en relief une approche * formelle * tant de la langue que de l'expression poétique, la première moitié de la séquence la donne comme peu pertinente. Alors que la règle proposée ne coïncide avec aucun usage réel du mot "hostie", l'analyse du vers, non seulement ne débouche pas sur une saisie éclairante du sens, mais scelle la futilité de ce genre d'analyse ${ }^{15}$. Nous n'avançons guère, et il s'est vraiment agi d'une "pêche à l'orignal " (p. 36).

Prenant en considération pareillement un fait « linguistique * et un fait "poétique», la deuxième moitié de la séquence ne les reprendra plus cette fois comme objet d'analyse, mais dans leur rapport à un sujet. C'est ainsi que rapportée à différents sujets et à différents contextes, la définition du mot "règles " est donnée comme ambiguë. Pour Chateaugué, le terme n'a qu'un sens, celui d'un outil de mesure, même si l'objet peut servir à "donner des fessées et à tirer des lignes n (p. 37). Mille Milles a par contre une tout autre idée du même mot: bien que divisées en douze comme celles de Chateaugué,

15 L'analyse du premier alexandrin est ponctuée par un « Enfin... " désinvolte et quatre lignes de digression. 
ses «règles» font appel à une division mens[tr]uelle plutôt que spatiale. Que sa vision "impure "des mots amène Mille Milles à se traiter sévèrement de laid, de sale et de méprisable (p. 37) nous importe moins, à ce moment-ci, que le fait même pour les mots d'avoir délaissé leur fonction d'objet dénotatif pour celle d'une connotation pour le sujet. Il y a tout lieu de croire que la « règle» de l'alexandrin, qui prescrit elle aussi une division en douze, devra à un moment donné subir le même sort.

D'autant que lorsque la noirceur de l'âme de Mille Milles aura activé la citation d'un second alexandrin, célèbre et identifié, celui-là ("C'est du Nelligan *) — «Mon âme est le donjon des mortels péchés noirs» (p. 37) -, sera encore sollicité un traitement de lecture tourné vers le sujet. Le commentaire par l'esthétique sera laissé pour compte - ${ } \mathrm{Ce}$ n'est ni génial, ni général, ni maréchal" — au profit d'une contrainte que valorise habituellement la prose - * Mais c'est clair» - et d'un décodage par la biographie présumée de Nelligan - -Nelligan était victime de la noirceur, de la crasse d'âme, de l'impuritude» -... étant entendu que la biographie de Mille Milles en est à peu près au même point. Ainsi, la lecture de l'alexandrin ne s'effectue qu'au prix de son inscription dans l'univers de la prose, et donc d'une forme de dénaturation générique.

On dira qu'il y a dans tout cela beaucoup de parodie, et requérant d'être lue à un niveau second sinon entre les lignes. Sans être dupe et vouloir prêter une portée trop générale à cet extrait, nous croyons que s'en dégage une forte propension du narrateur pour ce qu'on pourrait appeler une forme de «discohérence $» 16$.

Le dysfonctionnement est d'ailleurs évident, tant sur le plan linguistique que sur le plan littéraire. Dans le premier cas, le personnage codifie grammaticalement une unité discursive - «hostie» - dont l'ambiguïté réside essentiellement dans sa dimension connotative; alors qu'il conclut son analyse polysémique de «règles» sur un jugement de valeur ("règles" au sens de menstruations serait vulgaire, comme l'emploi de «ça» à la place de «cela") même si un tel constat

16 Jean Ricardou a jadis proposé l'utilisation de $\propto$ terme pour décrire le travail de Claude Simon dans Tryptique, dans Claude Simon. Colloque de Cerisy dirigé par Jean Ricardou, coll. a $10 / 18$ ", n 945, Paris, UGE, 1975, p. 25. 
de vulgarité aurait eu bien davantage de pertinence dans l'analyse du mot «hostie . Commentant les alexandrins, et alors qu'il reconnaît la beauté du premier à partir d'un examen objectif du signifiant sans parvenir au sens, Mille Milles « désacralise » le second en mettant l'accent sur la transparence du signifié. C'est dire que pour le personnage, le décodage de l'expression poétique passe par un décodage prosaïque, une mise en contexte assurée ici par le journal qu'il rédige. D'inaccessible dans la première moitié de l'extrait, la valeur d'éléments formellement similaires s'éclaire dans la seconde, grâce à l'ajustement de l'environnement subjectif de Nelligan à celui de Mille Milles.

Le langage poétique n'a donc ni sens ni portée esthétique en lui-même; seule une contextualisation (émotive et textuelle) lui fait prendre une valeur pour le sujet. En définitive, ni Mille Milles ni Nelligan ne sont des «hommes de lettres"; ce sont les lettres (et leurs règles) qui doivent devenir humaines ${ }^{17}$.

\section{La poésie écrite par la prose}

On peut se demander si cette mise en valeur de la lecture subjective exercée sur la poésie d'autrui doit servir de modèle dans l'appréciation des manifestations en apparence les plus poétiques du roman: les dix poèmes 18 qui le ponctuent à intervalles réguliers ${ }^{19}$. Fait assez surprenant, cette production, à notre connaissance, a suscité peu d'intérêt. Au moment de la parution, Alain Bosquet y avait retrouvé «de courts poèmes parfaits de forme et d'une gentille fraîcheur " 20 passant outre aux nombreuses irrégularités de forme comme de fond. Plus récemment, et n'hésitant pas à qualifier ces poèmes $d^{\prime}$ " essentiels ", Franf̧oise Laurent ${ }^{21}$ en commente

17 Voir la préface proprement dite du roman (p. 8, neuf lignes) que nous ne commentons pas faute d'espace, mais dont nous avons des raisons de croire qu'elle va dans le sens proposé ici. Elle s'achève par: « Je ne suis pas un homme de lettres. Je suis un homme".

18 Nous ne retenons pas ici la création en vers du chapitre 9: il s'agit de deux quatrains séparés par du texte, et surtout, syntaxiquement intégrés à l'anecdote, $\infty$ qui n'est le cas d'aucuine autre création du narrateur.

19 Cet equilibre a déjà été relevé par Yves Taschereau dans a Le vrai Nez qui voque ", Études françaises, Montréal, vol. 11, n® 3-4, octobre 1975, p. 320.

20 Le Monde, Paris, vol. XXIV, $n^{\circ} 6932,26$ avril 1967, p. 11.

21 Françoise Laurent, op. cit., p. 75. 
16

quelques vers pris un à un, sans tenter de définir pour cette production un espace spécifique.

Ces créations poétiques méritent pourtant d'être regardées de près. Même si à première vue et à la limite, l'ensemble pourrait sembler constituer une entité indépendante dont l'amputation laisserait l'ensemble-récit à peu près intact, il faut pourtant faire l'hypothèse d'une dialectique entre les deux. Très tôt à la lecture, les liens apparaissent d'ailleurs nombreux et à plusieurs niveaux. Le travail à faire, et que nous ne ferons pas ici, serait sans doute de reprendre chaque poème dans son environnement textuel, pour essayer d'en capter les résonances. Nous nous attacherons plutôt à présenter les poèmes en deux groupes dont les tendances respectives pourront donner quelques indications quant à leur fonction.

Les poèmes de la première partie, jusqu'à "Pan Ique " (p. 142-143) affichent tous un certain nombre d'irrégularités linguistiques très évidentes. Le premier texte, "Édimbourg en série (Assez ri, Édimbourg) » (p. 57), dont le titre comporte sa propre moquerie, ne laisse véritablement éclater son insolence qu'au dernier vers ${ }^{22}$ - «Au point où j'en suis jman balance " - où la reprise du mot «balance " dans une autre acception que celle des vers 14 et 15 ("Mon cœur balance") opère un brusque changement de ton et vient couper court aux mielleux "poussin chéri " et "pigeon volage " des deux premières strophes. Comme les parenthèses le faisaient pour le titre, le dernier vers oblige le lecteur à remettre en cause tout ce qui précède.

Ce phénomème sera également caractéristique des poèmes suivants. Le poème sans titre de la page 70 semble un prétexte (plus ou moins sérieux) à la recherche d'effets sonores :

Dans la vallée des verts poissons

Dans la marine des moissons

[...]

Une avalanche les avale

Sur le chemin de le cheval

Le fait pourtant d'orthographier certains mots des derniers vers sous la contrainte du nombre de pieds requis:

22 Voir aussi, un peu avant, le vers 10: « Mais plus ça tomb su le tapis ". 
Celleu où s'éteint la tendresse

Et avec ell le feu du drame

démasque l'ironie d'une dépendance excessive à la métrique. Quant à la finale de "Et l'herbe et le limon qui en pend" (p. 120):

Alors, alors, on vit qu'elle a-

Vait le derrièr plein de cendras,

elle vient désamorcer à son tour le reste du poème, dont elle interroge les enjeux.

Loin d'être des maladresses du narrateur, ces petites transgressions soulignent, en l'exacerbant, sa maîtrise des contraintes formelles du genre poétique: nous suggérons que le fait de la subversion pointe justement le travail sur le langage comme le principal (le seul?) propos de ces poèmes. Il faut dire d'ailleurs que ces premières créations manient une poésie tout embarrassée de stéréotypes: de l'exotisme et du ton vaguement moyenâgeux d' "Édimbourg en série ", aux prouesses d'allitérations du poème sans titre, puis au merveilleux incarné, dans «Et l'herbe et le limon qui en pend $»$, par la reine Bodale, femme bleue qui crache le feu. Aussi n'est-il pas étonnant que ces poèmes, à la limite de l'« exercice», apparaissent bien loin de l'histoire de Chateaugué et de Mille Milles, telle qu'elle est consignée dans le journal de celui-ci.

À partir de «La tour» (p. 158), une tendance générale des poèmes les accorde au contraire à la chronique rapportée, en même temps que disparaît toute impertinence formelle ou grammaticale (ces derniers poèmes sont sûrement ceux qui illustrent le mieux le commentaire d'A. Bosquet auquel nous avons déjà fait allusion). Les liens entre les poèmes et la prose sont parfois flagrants. «Loin de vous* (p. 167) intervient justement pendant l'absence de Chateaugué, au moment où le narrateur est hanté par le souvenir de celle-ci; même cas pour "Les automobiles" (p. 214-215) dont le sujet était apparu dès les premiers chapitres, lors d'un accident de Chateaugué, et dont la dénonciation est un thème récurrent du roman; comment ne pas voir également dans les deux vers qui terminent «Deux qui vont s'éteindre » (p. 235-236):

Regarde passer la corneille

Sous les noirs fils téléphoniques

une allusion à un épisode qui sera relaté quelques pages plus loin: «Une petite vieille est passée près de nous. Son manteau 
noir nous a frôlés; elle nous a effleurés avec ses ailes noires » (p. 256).

Le titre de ce poème renvoie d'ailleurs directement au projet de suicide des protagonistes. Si bien qu'il n'y a pas lieu de s'étonner si les thèmes du dernier poème, «Le dix-étages * (p. 268), recoupent presque exactement ceux de la proche anecdote: ce poème finit par n'être que la mise en vers des événements qui viennent d'être racontés (p. 264-266).

Bref, si les nombreux poèmes qui ponctuent le roman de Ducharme peuvent donner l'impression que la poésie commande la prose, on conviendra que cette forme poétique en vient à être complètement inversée, d'être envahie par un contenu prosaïque.

\section{Pour conclure}

Le nez qui voque représente une telle épreuve de décodage qu'il serait téméraire de prétendre en débusquer la signification dans le cadre d'un court article. Pour ramener notre bilan aux extraits étudiés, disons que la présence de la poésie semble prendre son sens dans une contradiction qui l'articule à la prose. C'est alors ce positionnement face à face des deux formes d'écriture, et partant leur double maintien, qu'il faut interroger.

Toute construction d'écriture ne peut se faire qu'à partir de ce qui existe déjà, et en même temps vise autre chose. Ce processus joue pour toutes les figures du monde qu'un texte emprunte nécessairement. Pour décrire ce phénomène, Louis Panier avait proposé il y a quelques années le concept de * recatégorisation * 23; plus récemment, il utilise, dans le même contexte, le couple * brouillage de sens/reconfiguration $* 24$.

C'est-à-dire que les *éléments signifiants * à partir desquels s'élabore le discours sont forcément brouillés, comme annulés, au départ, seule condition pour qu'ils puissent être redéfinis, recatégorisés, ou reconfigurés, « par leurs relations mutuelles dans le discours ${ }^{25}$. Sans ce travail chaque fois inédit, pas de compétence énonciative et pas d'écriture.

23 A.J. Greimas et J. Courtés, op. cit., p. 393.

24 Louis Panier, - Une lecture sémiotique des textes: questions de théologie biblique ", dans Sémiotique et Bible, décembre 1989, p. 25.

25 lbid. 
Avec les figures de la poésie, nous croyons avoir démontré que Le nez qui voque ne fait pas autre chose que ce travail. Articulant d'abord son propos sur une figure poétique empruntée à la littérature existante, le roman la brouille (notre partie I), ce qui mènera en bout de piste, si l'on veut, à une nouvelle forme de poème, totalement investie par l'anecdote du roman qu'on vient d'écrire (notre partie III). La différence entre les deux, et en même temps la condition du passage, réside, on l'aura compris, dans la référence obligée au Sujet (d'énonciation) de ce texte-ci (notre partie II). Au fond la poésie de Soir d'hiver et la prose du Nez qui voque sont dans le même rapport que le "cela» et le «ça» renvoyés nez à nez dans la «Préface»:

Quel est celui de ces deux pronoms démonstratifs qui est le meilleur: cela, ça ? Si c'est ça ce n'est pas cela et si c'est cela, ce n'est pas ça (p. 8) ${ }^{26}$.

Les deux sont d'autant plus compatibles et nécessaires qu'ils décrivent un même phénomène à des moments différents du processus d'écriture: si Le nez qui voque a pu paraître le "ça" de Soir d'hiver, il faut bien voir qu'il est aussi désormais, une fois scellé dans sa propre forme, un nouveau " cela *; et qu'en même temps, toute valorisation de l'un ou de l'autre genre est sans pertinence.

Disons les choses radicalement: Réjean Ducharme n'est pas un poète, et Le nez qui voque n'est pas un roman sur la poésie. Mais il est certes question, dans ce roman, à travers le passage d'une forme à une autre, de la production d'une écriture.

26 Sur ces pronoms, voir aussi les commentaires de Mille Milles (p. 37). 